\title{
SELF ORGANIZING MAP BASED IMAGE COMPRESSION USING CDF 9/7 LIFT DISCRETE WAVELET TRANSFORM
}

\author{
Aparna $\mathbf{K}^{\mathbf{1}}$, Arun Vikas Singh ${ }^{2}$ \\ ${ }^{1}$ M-Tech Student, Department of ECE, TJIT, Bengaluru, Karnataka, India \\ ${ }^{2}$ Head of the Department, ECE, TJIT, Bengaluru, Karnataka, India
}

\begin{abstract}
In this paper a new method for image compression using Self Organizing Map and CDF $9 / 7$ lift Discrete Wavelet Transform is proposed. Here Self Organizing Map is used to generate the code vectors associated with the input data there by reducing the dimension of the input data. Discrete Cosine Transform is used in the existing methods of image compression which results in the degradation of the reconstructed image. Hence to improve the reconstructed image quality $9 / 7$ lift DWT is used. Peak Signal to Noise Ratio (PSNR) and Compression ratio (CR) is calculated for various images using the proposed method and they show better results than the existing methods.
\end{abstract}

KEYWORDS: Self Organizing Map (SOM), CDF Lift DWT, Peak Signal To Noise Ratio (PSNR), Compression Ratio $(C R)$.

\section{INTRODUCTION}

Image compression is a technique that reduces the number of bits used to represent an image ie, the redundant bits of an image is discarded. But during this redundancy removal process loss of any information is not acceptable. Compression of image can be classified in to two categories:-lossy image compression and lossless image compression. There is no loss of information during lossless image compression and original data can be reconstructed. Therefore in applications such as medical field, lossless image compression is used. In such applications exact reconstruction of the data should be possible. High values of performance parameters such as PSNR and CR is possible in lossy image compression. Here also the original data can be reconstructed but it consists of certain degradation. Lossy image compression can achieve tremendous reduction in transmission bandwidth, storage cost and storage space. Existing techniques for image compression used Discrete Cosine Transform (DCT) and DWT, out of which DWT has many advantages compared to DCT. Neural network also play an important role in image compression. Different types of neural networks include feed forward neural network, back propagation neural network, Self organizing Map etc. Vector quantisation is an efficient method for image compression and it provides high $\mathrm{CR}$ and PSNR. They use residual code book and indices for image compression. Residual codebooks are used for improving the quality of the reconstructed image by reducing the distortions in the compressed images. Even though vector quantisation based image compression provides better PSNR and CR there is a disadvantage associated with this technique, ie computational time and computational complexity is very high. But SOM which is a type of Artificial neural Network used in this approach resolved the drawbacks of vector quantisation.
In this paper image compression using SOM and CDF 9/7 lifting DWT is explained. SOM achieves image compression by generating the code vectors associated with the input data. The advantage of lifting based DWT compared to convolution DWT is that the former has better reconstruction property and computation time is also lesser. Remaining of this paper is arranged as section 2 describes the literature survey, section 3 describes the proposed methodology, section 4 analyses the result and section 5 concludes the paper.

\section{LITERATURE SURVEY}

Sathappan [1] proposed image compression technique based on vector quantisation and residual code book .Here compression is mainly achieved by compressing the indices of vector quantisation. A residual codebook decreases the distortion in the reconstructed image and hence provide high peak signal to noise ratio(PSNR) value.

Yusong $\mathrm{Hu}$ and Ching Chuen Jong [2] presented a parallel lifting based 2D-DWT architecture with high memory and short critical path .Compared to conventional based DWT, lifting based DWT requires less memory efficiency. A trade off between external memory band width and on-chip memory which is achieved through parallel stripe based data scanning provides memory efficiency. Another advantage of this technique compared to other existing technique is that hardware requirement and computation complexity is less for this method.

Vipula Singh et al. [3], introduced an image compression method using fuzzy vector quantisation, neural network and wavelet transform. Here the processing speed of the image can be increased by dividing the image in to different sub bands and then each sub bands is processed parallely. The 
results of the proposed method is compared with JPEG and JPEG2000 and proved that the proposed method is more efficient. Also this method is capable of handling more noise. This is because that neural network and vector quantizer is capable of filtering noise from the image distorted with noise.

Pacha Sreenivasulu et al. [4], proposed a hybrid technique for compressing an image with free run length coding as well as wavelet transformation. This method provides good compression with very good visual quality.

Christophe Amerijckx et al. [5], presented an idea for compressing image with the help of Kohonen SOM and Discrete Cosine Transform. The algorithm for SOM creates relationship among input space and output space. When the result of this proposed method is compares with JPEG compression, it is seen that the proposed method provides better compression ratio and visual quality.
G.Muthulakshmi et al.[6], introduced a new method for image compression using code book generation with SOM and wavelet transform. Here the wavelet coefficients obtained from the DWT is converted in to Vector Quantisation code vectors. Better Compression Ratio, Peak Signal to Noise Ratio and image quality is obtained by the proposed method.

\section{PROPOSED METHODOLOGY}

In this section, the image compression using Self Organizing Map and CDF 9/7 lift DWT is described. This hybrid technique is used to achieve better compression ratio and high reconstructed image quality.

The block diagram for compression and reconstruction of an image is given in figure 1 and figure 2 .

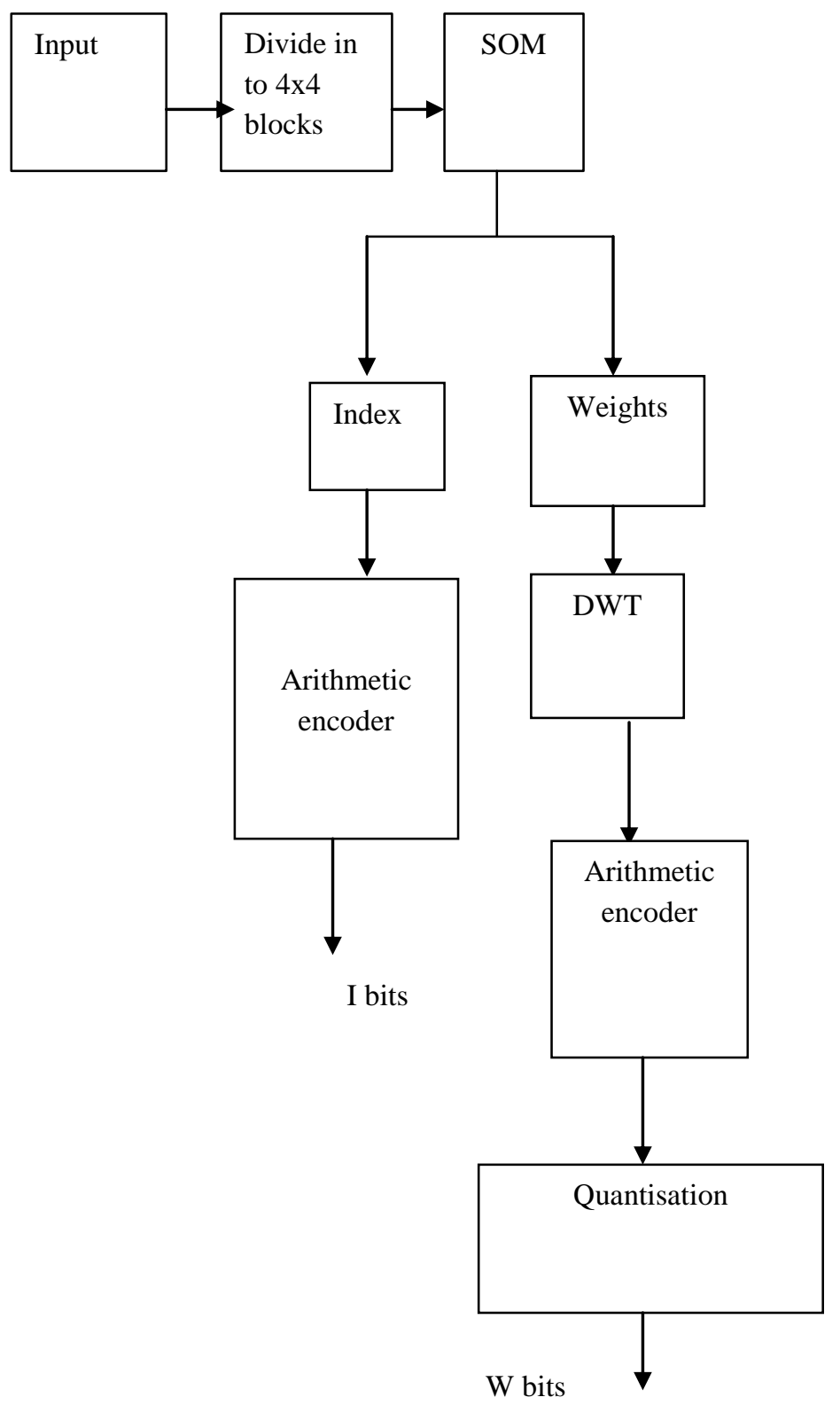

Fig.1 Block diagram for compression 


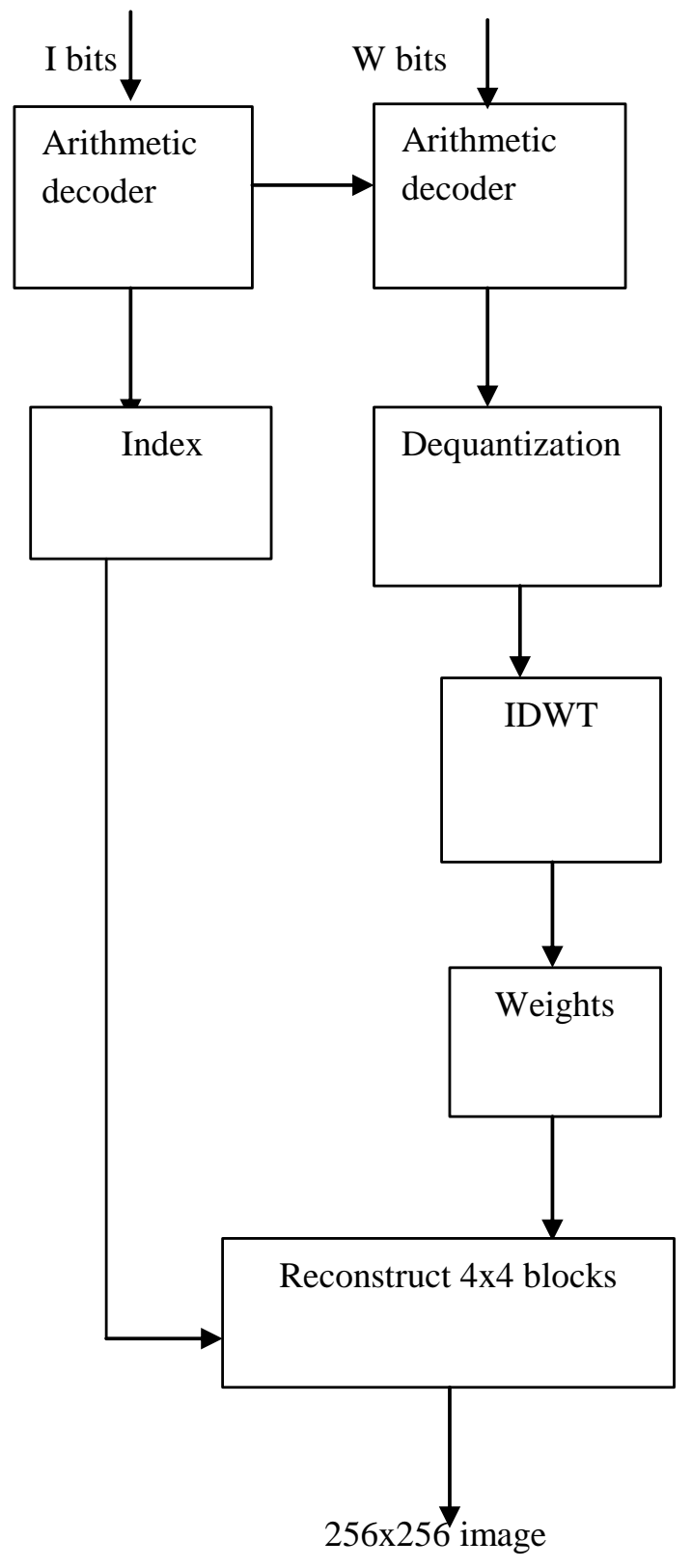

Fig: 2 Block diagram for reconstruction

\subsection{Self Organizing Maps}

Self Organizing Map is a classification under artificial neural network. It is an unsupervised training system based on competitive learning. The difference between supervised and unsupervised training system is that, for a supervised training system there will be a target and for an unsupervised training system there will not be any target and network learn to classify the input vectors among themselves. The main aim of a SOM is to convert an input signal of higher dimension in to a lower dimension signal. The SOM is set up by placing neurons at the nodes of the lattice. Associated with each neuron there will be an index value and weight value. Four important steps in the setting up of SOM includes initialization, competition, co operation and adaptation. The output of the SOM will be code vectors associated with the input data. Thus SOM helps in the compression of data.

\subsection{9/7 Lift Dwt}

Discrete Wavelet Transform (DWT ) is another method for image compression. When DWT is applied to an image different sub band coefficients of the image is obtained. The sub band coefficients are mainly approximation co efficient and (LL) and detailed coefficients (LH, HL and HH). The implementation of discrete wavelet can be done in two ways :- convolution based approach and lifting based approach. In this proposed method lifting based $9 / 7$ is used. $9 / 7$ is the representation of filter coefficients. Compared to convolution based DWT 9/7 lift DWT has better performance and computational complexity is less. Reconstruction property is also better for lifting based DWT when compared to convolution DWT.

The 3 steps involved in the lifting based DWT are Splitting, Lifting and Scaling

Splitting:- Input data is divided in to even and odd terms based on their position.

$\mathrm{Xe}=\mathrm{X}(2 \mathrm{i})$

$\mathrm{Xo}=\mathrm{X}(2 \mathrm{i}+1)$

Lifting:- Lifting has 2 stages prediction and updation. Prediction and updation is done 2 times in 9/7 lift DWT.

Predict $\mathrm{P} 1: \mathrm{D} 1(\mathrm{i})=\mathrm{Xo}(\mathrm{i})+\alpha[\mathrm{Xe}(\mathrm{i})+\mathrm{Xe}(\mathrm{i}+1)]$

Update U1:S1(i) $=\mathrm{Xe}(\mathrm{i})+\beta[\mathrm{D} 1(\mathrm{i}-1)+\mathrm{D} 1(\mathrm{i})]$

Predict P2: D2(i) $=\mathrm{D} 1(\mathrm{i})+\gamma[\mathrm{S} 1(\mathrm{i})+\mathrm{S} 1(\mathrm{i}+1)]$

Update U2:S2(i) $=\mathrm{S} 1(\mathrm{i})+\delta[\mathrm{D} 2(\mathrm{i}-1)+\mathrm{D} 2(\mathrm{i})]$

$\alpha=-1.506134342$

$\beta=-0.0529801185$

$\gamma=0.882911076$

$\delta=-0.443506852$

$\mathrm{k}=1.149604398$

Scaling:- Scaling is the process of normalisation of filter coefficients.

$\mathrm{YH}(\mathrm{i})=1 / \mathrm{k} * \mathrm{D} 2(\mathrm{i})$

$\mathrm{YL}(\mathrm{i})=\mathrm{k} * \mathrm{~S} 2(\mathrm{i})$

\subsection{Implementation}

Steps involved for compression and reconstruction of the image in this approach is given below.

\subsubsection{Compression}

The input image of size $256 \times 256$ is divided in to $4 \times 4$ blocks (4096 such blocks are obtained) which are then converted in to $16 x 4096$ vectors. Then the $16 \times 4096$ vectors are fed as an input to SOM to get 16X256 weight matrix and 4096 index values. The index values are then arithmetically encoded and saved as I bits. 9/7 DWT is applied to weight matrix and the different sub band coefficients obtained are encoded and quantised and stored as $\mathrm{W}$ bits.

\subsubsection{Reconstruction}

The I bits are decoded to get back the index values. The $\mathrm{W}$ bits are decoded and dequantised and the IDWT is applied to get back the weight matrix. The 16x4096 vectors are reproduced from the index values and weight matrix and then it is converted back to $4 \times 4$ sub blocks. Finally $256 \times 256$ image is obtained as output. 


\section{RESULT AND ANALYSIS}

The proposed method is tested for various images like Lena, cameraman, mandrill and pepper. PSNR and CR values for these images are calculated and listed in the table below. High values of PSNR and CR are obtained for these images using the proposed approach compared to existing techniques
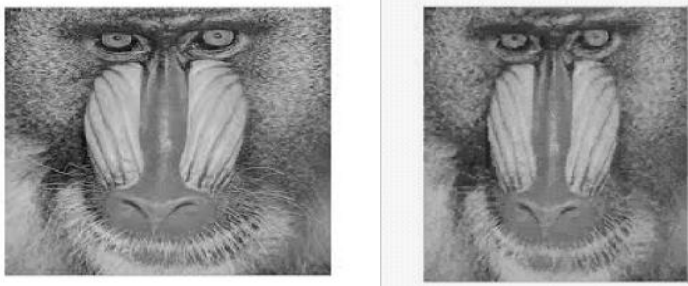

Fig 3. Mandrill original and reconstructed images

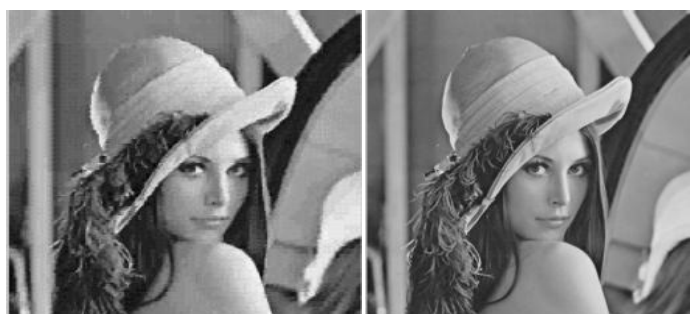

Fig 4. Lena original and reconstructed images

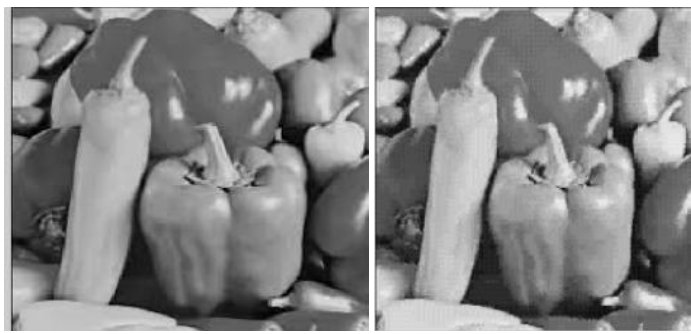

Fig 5. Pepper original and reconstructed images

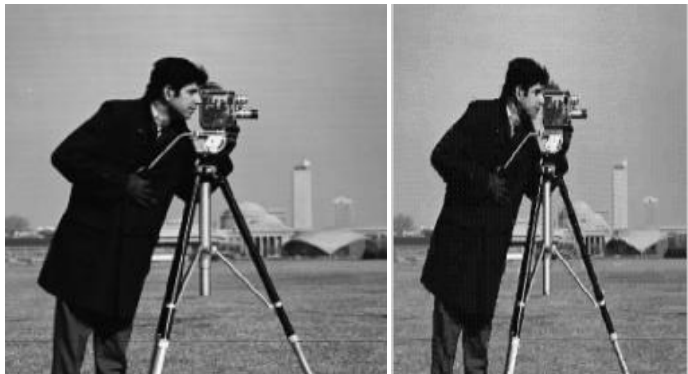

Fig 6. Cameraman original and reconstructed images

Table 1. PSNR and CR values of different images obtained using the proposed method.

\begin{tabular}{|l|l|l|}
\hline Images & PSNR & CR \\
\hline Mandr ill & 28.02 & 13.661 \\
\hline Lena & 29.48 & 12.41 \\
\hline Pepper & 30.274 & 12.357 \\
\hline Cameraman & 27.726 & 12.317 \\
\hline
\end{tabular}

\section{CONCLUSION}

The paper can be concluded by saying that the proposed method using Self Organizing Map and 9/7 Lift Discrete Wavelet Transform achieves higher values of Compression Ratio and Peak Signal to Noise Ratio. SOM also plays a major role in image compression by generating the code vectors. 9/7 Lift DWT has the property of best reconstruction when compared to convolution DWT. Hence in this proposed method quality of the reconstructed image is very good.

\section{REFERENCES}

[1]. Dr.S.Sathappan,"A Vector Quantisation Technique for Image Compression using Modified Fuzzy Possibilistic C-Means with Weighted Mahalanobis Distance",International Journal of Innovative Research in Computer and Communication Engineering,vol.1,issue 1,pp.12-20,March 2013

[2]. Yusong $\mathrm{Hu}$ and Ching Chuen Jong,"A MemoryEfficient Scalable Architecture for Lifting Based Wavelet Transform",IEEE Transaction on Circuits and Systems,vol.60,no.8,pp.502-506,August 2013.

[3]. Vipula singh, Navin Rajpal and k.Srikanta Murthy,"An Algorithmic Approach for Efficient Image Compression using Neuro Wavelet Model and Fuzzy Vector Quantisation Technique, International Journal on Computer Science and Engineering,vol.2,n0.07,pp.2366-2374,2010.

[4]. Pacha Sreenivasulu and Kancharla Anitha sheela,"A Novel Wavelet Based Approach for Lossless Image Compression using Modified Duplicate Free Run Length Coding", Journal of Theoretical and Applied Computer Science,vol.8.n0.3,pp.51-57,2014.

[5]. Christophe Amerijckx and Philippe Thissen,"Image Compression by Self Organized Kohonen Map", IEEE Transactions of Neural Networks,vol.9,no.3,pp.503507.May 1998.

[6]. G.Muthu Lakshmi and V.Sadasivam,'Image Compression Using Self Organizing Feature Map and Wavelet Transformation", ICTAT Journal on Image and Video Processing,vol.3,issue.1,pp.445-451,August 2012 .

\section{BIOGRAPHIES}

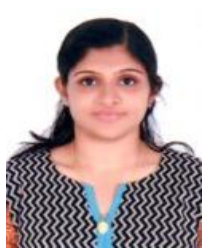

Ms. Aparna $\mathbf{K}$ is pursuing $\mathrm{M}$-tech degree in Digital Communication and Networking from T-John Institute of Technology, Bengaluru affliated to Visvesvaraya Technological University.

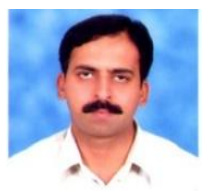

Dr. Arun Vikas Singh is the Head of the Department of Electronics and Communication Engineering, T-John Institute of Technology, Bengaluru. 